Pacific Journal of Mathematics

CONCERNING CERTAIN LOCALLY PERIPHERALLY 


\section{CONCERNING CERTAIN LOCALLY PERIPHERALLY SEPARABLE SPACES}

\section{B. TREYBig}

In 1954, F. Burton Jones raised the question [2] "Is every connected, locally peripherally separable [3], metric space separable?"' In this paper it will be shown that there exists a connected, semi-locally-connected, space $\Sigma$ satisfying R. L. Moore's axioms 0 and $C^{1}$, in which every region has a separable boundary, every pair of points is a subset of some separable continuum ${ }^{2}$, and the set of all points at which $\Sigma$ is not locally separable is separable. It will also be shown that every compactly connected, locally peripherally separable, metric space is completely separable.

\section{PART 1}

Let $S^{\prime}$ denote the set of all points of the Euclidean plane $E$. A square disk in $E$ will be said to be horizontal if it has two horizonta sides. A point set in $E$ will be called an $H$-disk only if that set is a horizontal square disk. By the width of a square disk will be meant the length of one of its sides.

Let $K$ denote a definite $H$-disk of width $d$. Let $R_{0}(K)$ denote the $H$-disk of width $d / 4$ whose center is on the vertical line that contains the center of $K$, and whose upper side lies at a distance of $d / 16$ below the upper side of $K$. Let $R_{00}(K)$ and $R_{01}(K)$ denote the $H$-disks of width $d / 8$ whose upper sides are at a distance of $d / 32$ above the lower side of $R_{0}(K)$ and whose centers are on the vertical lines containing the left and right sides, respectively, of $R_{0}(K)$.

In general, for each positive integer $n$ let $U_{n}(K)$ denote a collection of $2^{n}$ mutually exclusive congruent $H$-disks such that

(1) $R_{01}(K)$ and $R_{00}(K)$ are the elements of $U_{1}(K)$,

(2) if $n$ is a positive integer and $y$ is an element of $U_{n}(K)$, and $x$ and $z$ are $H$-disks of width $d / 4(2)^{n+1}$ whose centers lie on the same vertical lines as the left and right sides of $y$, respectively, and whose upper sides lie at a distance of $d / 32(2)^{n}$ above the lower side of $y$, then $x$ and $z$ are elements of $U_{n+1}(K)$.

If $n$ is a positive integer and $R_{x_{1} x_{2} \cdots x}(K)$ is an elements of $U_{n}(K)$, then let the elements $x$ and $y$ of $U_{n+1}(K)$ whose centers lie on the same

Presented to the American Mathematical Society, June 15, 1957; received by the editors September 4, 1958. This paper is part of a dissertation submitted to the Graduate School of the University of Texas in partial fulfillment of the requirements for the $\mathrm{Ph}$. D. degree.

1 The proof that every space which satisfies axioms 0 and $C$ is metric is due to R. L. Moore.

2 A continuum is a connected, closed set. 
vertical lines as the left and right sides of $R_{x_{1} x_{2} \cdots x_{n}}(K)$, respectively, be denoted by $R_{x_{1} x_{2} \cdots n_{n^{0}}}(K)$ and $R_{x_{1} x_{2} \cdots x_{n^{1}}}(K)$, respectively. Let $C(K)$ be a collection to which $x$ belongs if and only if $x$ is $R_{0}(K)$ or in one of the collections $U_{1}(K), U_{2}(K), \cdots$.

Let $L(K)$ denote the $H$-disk of width $d / 8$ whose center is on the same vertical line as the center of $K$, and whose lower side is at a distance of $3 d / 16$ above the lower side of $K$. Let $P_{l}(K)$ and $P_{r}(K)$ denote the left and right-hand end points, respectively, of the lower side of $L(K)$. Let $M(K)$ denote the point set such that a point $P$ belongs to it if and only if $P$ is a point of the interval $P_{l}(K) P_{r}(K)$ such that there is no nonnegative integer $p$ and positive integer $q$ such that $P P_{l}(K) / P_{l}(K) P_{r}(K)=p_{i} / 2^{\alpha}$. Let $I(K)$ denote the collection to which $x$ belongs if and only if $x$ is a vertical interval containing a point of $M(K)$, and with both end points on the boundary of $L(K)$. Let an interval $i$ of $I(K)$ be denoted by $i_{x}(I(K))$ if and only if it is true that if $P$ is the lowest point of $i$, then $P_{l}(K) P / P_{l}(K) P_{r}(K)=x$.

Let $R$ denote some definite $H$-disk. Let $R_{0}(R)$ be denoted by $Q_{0}$; let $R_{00}(R)$ and $R_{01}(R)$ be denoted by $Q_{00}$ and $Q_{01}$, respectively. Let $R_{000}(R), R_{001}(R), R_{010}(R)$, and $R_{011}(R)$ be denoted by $Q_{000}, Q_{001}, Q_{010}$, and $Q_{011}$, respectively, and so forth. Let $C(R)$ be denoted by $C_{1}$ and let $I(R)$ be denoted by $I_{0}$.

Let $C_{2}$ denote the collection to which $x$ belongs if and only if $x$ is an element of $C(y)$, for some element $y$ of $C_{1}$ distinct from $Q_{0}$. Let $R_{0}\left(Q_{00}\right)$ be denoted by $Q_{00,0}$; let $R_{01}\left(Q_{00}\right)$ be denote by $Q_{00,01}$. In general, let $R_{x}\left(Q_{y}\right)$ be denoted by $Q_{y, x}$. Also, if $Q_{x}$ is in $C_{1}$ and $x \neq 0$, let $I\left(Q_{x}\right)$ be denoted by $I_{x}$.

In general, let $C_{n+1}$ denote the collection to which $x$ belongs if and only if $x$ is an element of $C(y)$, for some elements $y$ of $C_{n}$, which, in case $x_{n}$ is 0 , is distinct from $Q_{x_{1}, x_{2}, \ldots, x_{n}}$. Let the element $R_{x_{n+1}}\left[R_{x_{n}}\left[R_{x_{n-1}}\left[\cdots\left[R_{x_{1}}(R)\right] \cdots\right]\right]\right]$ of $C_{n+1}$ be denoted by $Q_{x_{1}, x_{2}, \ldots, x_{n+1}}$. Also if $w$ is the element $Q_{x_{1}, x_{2}, \cdots, x_{n}}$ of $C_{n}$ and $x_{n} \neq 0$, then let $I(w)$ be denoted by $I_{x_{1}, x_{2}, \ldots, x_{n}}$. For each $n$ let $I_{n}$ be the collection to which $x$ belongs if and only if there is an element $Q_{x_{1}, x_{2}, \ldots, x_{n}}$ of $C_{n}$ such that $x_{n} \neq 0$ and $x$ is in $I\left(Q_{x_{1}, x_{2}, \ldots, x_{n}}\right)$.

Let $W$ denote the point set to which a point $P$ belongs if and only if $P$ belongs to $C_{n}^{* 3}$ for each positive integer $n$. For each positive integer $n$ let $B_{n}$ denote the collection of all the boundaries of the elements of $C_{n}$. The boundary of $Q_{x_{1}, x_{2}, \cdots, x_{n}}$ will be denoted by $J_{x_{1}, x_{2} \cdots, x_{n}}$.

Let $S$ denote $\left[I_{0}^{*}+I_{1}^{*}+\cdots\right]+\left[B_{1}^{*}+B_{2}^{*}+\cdots\right]+W$.

Let $C^{\prime}$ be a collection to which $w$ belongs if and only if $w$ is $R$ or $I(w)$ is a subset of $S$ and there is a positive integer $n$ such that $w$ is in $C_{n}$.

\footnotetext{
${ }^{3} C_{n}^{*}$ Means the sum of all the point sets of the collection $C_{n}$.
} 
For each positive integer $n$ let $H_{n}$ denote a collection to which $x$ belongs if and only if $x$ is the common part of $S$ and the interior of some square of $\left[B_{n}+B_{n+1}+\cdots\right]$. For each element $Q_{x_{1}, x_{2} \cdots, x_{n}}$ of $C_{n}$, let the set of all points of $S$ in the interior of $J_{x_{1}, x_{2}, \cdots, x_{n}}$ be denoted by $r_{x_{1}, x_{2}, \ldots, x_{n}} \cdot$

For each positive integer $n$ let $K_{n}$ denote a collection to which $x$ belongs if and only if, either (1) $x$ is a segment of an arc lying on some square $J$ of $\left(B_{1}+B_{2}+\cdots\right)$, having length less than $1 / 4^{n}$ times the perimeter of $J$, and intersecting no square of the collection $\left(B_{1}+B_{2}+\cdots\right)$ except $J$, or (2) $x$ is the sum of two straight line segments $p$ and $q$ intersecting at their midpoints and lying on different squares $J_{p}$ and $J_{q}$ of $\left(B_{1}+B_{2}+\cdots\right)$, such that $p$ and $q$ each have length less than $1 / 4^{n}$ times the perimeters of $J_{p}$ and $J_{q}$, respectively, and such that neither $p$ nor $q$ intersects three squares of $\left(B_{1}+B_{2}+\cdots\right)$.

Suppose $x$ is a positive number such that $i_{x}\left[I_{j_{1}, j_{2}, \ldots, j_{n}}\right]$ is an interval of $I_{j_{1}, j_{2}, \ldots, j_{n}}$. For each positive integer $n$ there exists a unique pair $\left(k_{n}, x_{n}\right)$ such that $k_{n}$ is a non-negative integer, $x_{n}$ is a positive number less than one, and $x=\left(k_{n}+x_{n}\right) / 2^{n}$. By $i_{n}\left[i_{x}\left(I_{j_{1}, j_{2}, \ldots, j_{n}}\right)\right]$ will be meant the vertical interval $i_{x_{n}}(I(y))$, where $y$ is the $H$-disk of $U_{n}\left[Q_{j_{1}, f_{2}, \ldots, j_{n}}\right]$ with only $k_{n}$ disks of $U_{n}\left(Q_{j_{1}, j_{2}, \ldots, j_{n}}\right)$ to the left of it.

Suppose, for some $y$ in $C^{\prime}, P$ is the highest point of the interval $i_{x}(I(y))$. By $R_{n}(P)$ will be meant the sum of all the sects $z$ such that either

(1) for some positive integer $d$ greater than or eqal to $n, z$ is the subset of $i_{a}\left[i_{x}(I(y))\right]$ with length $1 / 2^{n}$ times the length of $i_{a}\left[i_{x}(I(y))\right]$ that contains the lowest point of $i_{a}\left[i_{x}(I(y))\right]$, or

(2) $z$ is the subset of $i_{x}[I(y)]$ with length $1 / 2^{n}$ times the length of $i_{x}(I(y))$ that contains the highest point of $i_{x}(I(y))$.

For each positive integer $n$ let $L_{n}$ denote a collection such that $x$ belongs to it if and only if there exists a positive integer $d$ greater than or equal to $n$, an element $y$ of $C^{\prime}$, and an interval of the collection $I(y)$ such that if $P$ denotes the highest point of that interval, then $x=R_{a}(P)$.

For each positive integer $n$ let $N_{n}$ denote a collection to which $x$ belongs if and only if either

(1) for some element $y$ of $C^{\prime}$ there exists an interval $i$ of the collection $I(y)$ such that $x$ is a segment of $i$ of length less than $1 / 2^{n}$ times the length of $i$, or

(2) for some element $y$ of $C^{\prime}$ there exists an element $i$ of $I(y$, such that $x$ is a sect lying in $i$, containing the lowest point of $i$ and of length less than $1 / 2^{n}$ times the length of $i$.

For each positive integer $n$ let $G_{n}$ denote a collection to which $x$ belongs if and only if it lies in $H_{n}+K_{n}+L_{n}+N_{n}$. $S$ is the set of 
all points of $\Sigma$. A subset $r$ of $S$ is a region in $\Sigma$ if and only if $r$ belongs to $G_{1}^{4}$.

R. L. Moore's axioms 0 and $C$ are as follows:

Axiom 0. Every region is a point set.

Axiom $C$. There exists a sequence $G_{1}, G_{2}, \cdots$ such that

(1) for each positive integer $n, G_{n}$ is a collection such that each element of $G_{n}$ is of region and $G_{n}$ covers $S$,

(2) for each $n, G_{n+1}$ is a subcollection of $G_{n}$,

(3) if $A$ is a point, $B$ is a point and $R$ is a region containing $A$, then there exists a positive integer $n$ such that if $x$ is a region of $G_{n}$ containing $A$ and $y$ is a region of $G_{n}$ intersecting $x$, then

(a) $y$ is a subset of $R$ and

(b) if $B$ is not $A, y$ does not contain $B$,

(4) if $M_{1}, M_{2}, \ldots$ is a sequence of closed point sets such that for each $n$ there exists a region $g_{n}$ of $G_{n}$ such that $M_{n}$ is a subset of $\bar{g}_{n}$ and for each $n M_{n}$ contains $M_{n+1}$, then there is a point common to all the point sets of this sequence.

It is obvious that in the space $\Sigma$ each region has a countable, and therefore separable, boundary, and that the sequence $G_{1}, G_{2}, \ldots$ defined for the space $\Sigma$ satisfies conditions (1) and (2) of axiom $C$. It will be shown that it also satisfies conditions (3) and (4) of this axiom.

Suppose that $P$ is a point of $W$, that $r=r_{x_{1}, x_{2}, \ldots, x_{n}}$ is a region of $H_{n}$ containing $P$, and that $Q$ is a point of $r$ distinct from $P$. If $q$ is a region containing a point of $W$, then $q$ must belong to $H_{1}$. Since each element of $C_{n+1}$ which contains $P$ has a side of length less than or equal 1/4 times the length of a side of $Q_{x_{1}, x_{2}, \cdots, x_{n}}$, and each element of $C_{n+2}$ which contains $P$ has a side of length less than or equal $1 / 4^{2}$ times the length of side of $Q_{x_{1}, x_{2}, \ldots, x_{n}}$, and so forth; it is obvious that there is a $d>n$ such that if $q$ is a region of $H_{d}$ which contains $P$, then $\bar{q}$ does not intersect $Q$ and is a subset of $r$. Suppose that $x$ and $y$ are two intersecting regions of $G_{n+1}$ such that $x$ contains $P$. $x$ belongs to $H_{n+1}$ and is therefore a subset of $r$. Every region of $G_{n+1}$ which intersects $x$ is a subset of $r$, so clearly, $y$ is a subset of $r$.

Now suppose that $P$ is a point of $J_{x_{1}, x_{2}, \ldots, x_{n}}$ of $B_{n}$ and $r$ is a region containing $P$, and $Q$ is a point of $r$ distinct from $P$. There exists a circle $J$ in $E$ with center at $P$ such that every point of $S$ in the interior of $J$ belongs to $r$, but $Q$ is not in the interior of $J$. There exists a positive integer $d$ such that $1 / 4^{a}$ times the perimeter of any square of $\left(B_{1}+B_{2}+\cdots\right)$ to which $P$ belongs is less than the radius of $J$, and such that no region of $H_{d}$ contains $P$. If $R^{1}$ is a region of $G_{d+1}$ containing $P$, then $\bar{R}^{1}$ does not contain $Q$ and is a subset of $r$. If $n>d+2$

${ }^{4}$ The collection $G_{1}$ of regions is a basis for the space $\Sigma$. 
and $x$ and $y$ are two intersecting regions of $G_{n}$ such that $x$ contains $P$, then $x+y$ is a subset of $r$.

Now suppose that $P$ is a point of $i_{x}(I(y))$, for $y$ in $C^{\prime}$, and that $r$ is a region containing $P$ and that $Q$ is a point of $r$ distinct from $P$.

Case 1. Suppose $P$ is not the highest point of $i_{x}(I(y))$. There exists a segment $t$ containing $P$, or a sect in case $P$ is the lowest point of $i_{x}(I(y))$, such that $t$ is a subset of $r$ and does not contain $Q$ nor the highest point of $i_{x}(I(y))$. There exists a positive number $\varepsilon$ such that every point of $i_{x}(I(y))$ which is at a distance from $P$ of less than $\varepsilon$ lies in $t$. There exists a positive integer $d$ such that

(1) no region of $L_{d}$ intersects $t$ and no region of $H_{d}$ intersects $i_{x}(I(y))$, and

(2) $1 / 2^{d}$ times the length of $i_{x}(I(y))$ is less than $\varepsilon$. Therefore, if $k$ is a region of $G_{a+1}$ containing $P$, then $\bar{k}$ is a subset of $r$ and does not contain $Q$. Also, if $x$ and $y$ are two intersecting regions of $G_{a+2}$ such that $x$ contains $P$, then $x+y$ is a subset of $r$.

Case 2. Suppose $P$ is the highest point of $i_{x}(I(y))$. Whether $Q$ belongs to $i_{x}(I(y))$ or there is a positive integer $p$ such that $Q$ belongs to $i_{p}\left[i_{x}(I(y))\right]$ or $r$ is in $H_{1}$ and $Q$ does not belong to $\left.i_{x}(I(y))+i_{1}\left[i_{x}(I)\right)\right]+$ $i_{2}\left[i_{x}(I(y))\right]+\cdots$, there is a positive integer $d$ such that

(1) $R_{a}(P)$ does not contain $Q$ and is a subset of $r$, and

(2) no region of $H_{d}$ contains $P$. If $k$ is a region of $G_{d+1}$ containing $P$, then $\vec{k}$ is a subset of $r$ and does not contain $Q$. Also, if $x$ and $y$ are two intersecting regions of $G_{d+3}$ such that $x$ contains $P$, then $x+y$ is a subset of $r$.

Therefore $G_{1}, G_{2}, \ldots$ satisfies the third part of axiom $C$.

Suppose that $M_{1}, M_{2}, \cdots$ is a sequence of closed point sets such that

(1) for each $n M_{n}$ contains $M_{n+1}$, and

(2) for each $n$ there is a region $g_{n}$ of $G_{n}$ such that $M_{n}$ is a subset of $\bar{g}_{n}$.

In case, for each $n, g_{n}$ is in $H_{n}$, then by definition of $W$, there is a point common to $M_{1}, M_{2}, \cdots$ because some point of $W$ can be easily shown to be a limit point or point of $M_{n}$ for each $n$.

In case there is a positive integer $j$ such that $g_{j}$ belongs to $K_{j}$, then for $n>j, g_{n}$ belongs to $K_{n}$. But $M_{j}, M_{j+1}, \cdots$ is a sequence of closed and compact point sets such that for $n \geqq j M_{n}$ contains $M_{n+1}$. So there is a point common to $M_{j}, M_{j+1}, \cdots$ and thus common to $M_{1}, M_{2}, \cdots$.

In case there is a positive integer $j$ such that $g_{j}$ belongs to $N_{j}$, then for $n>j, g_{n}$ belongs to $N_{n}$. So, for the same reason as in the 
previous case, there is a point common to $M_{1}, M_{2}, \cdots$.

The only case not considered is the one where there is a positive integer $j_{1}$ such that, for $n \geqq j_{1}, g_{n}$ belongs to $L_{n}$. In this case $g_{j_{1}}$ must be $R_{x_{1}}(P)$ for some point $P$ and positive integer $x_{1}$. There is a positive integer $j_{2}>j_{1}$ such that $g_{j_{2}}=R_{x_{2}}(P)$, where $x_{2}>x_{1}$. There is a positive integer $j_{3}>j_{2}$ such that $g_{j_{3}}=R_{x_{3}}(P)$, for $x_{3}>x_{2}$, and so forth. $P$ is common to the sets $R_{x_{1}}(P), R_{x_{2}}(P), \cdots$. But if $P$ does not belong to each of the sets $M_{j_{1}}, M_{j_{2}}, \ldots$ then there is a positive integer $d$ such that $\bar{R}_{x_{a}}(P)$ contains no point of $M_{x_{j}}$ for any $j$. But $R_{x_{d}}(P)$ contains $M_{j_{d+1}}$. So $P$ is common to the sets $M_{j_{1}}, M_{j_{2}}, \cdots$ and thus common to $M_{1}, M_{2}, \cdots$.

Thus, $\Sigma$ satisfies the fourth part of axiom $C$.

In order to show that $\Sigma$ is connected, an indirect argument will be used. Suppose that $S$ is the sum of two mutually separated sets $H$ and $K$. Since $W+\left(B_{1}^{*}+B_{2}^{*}+\cdots\right)$ is connected, let $H^{\prime}$ be the one of the sets $H$ and $K$ that contains this set and let $K^{\prime}$ be the other. There exists an element $y$ of $C^{\prime}$ such that for some $x i_{x}[I(y)]$ is a subset of $K^{\prime}$. But there exists a positive integer $d_{1}$ such that for $n \geqq d_{1}$, $i_{n}\left[i_{x}(I(y))\right]$, belongs to $K^{\prime}$. There exists a positive integer $d_{2}$ such that for $n \geqq d_{2} i_{n}\left[i_{a_{1}}\left(i_{x}(I(y))\right)\right]$ belongs to $K^{\prime}$. So, obviously, there is a positive integer sequence, $d_{1}, d_{2}, \cdots$ such that if $j$ is a positive integer and $n \geqq d_{j}$, then $i_{n}\left(i_{a_{j-1}}\left(i_{a_{j-2}}\left(\cdots i_{a_{1}}\left(i_{x}(I(y))\right) \cdots\right)\right)\right)$ belongs to $K^{\prime}$. But from this fact it is easily seen that some point of $W$ is a limit point of $K^{\prime}$. So $\Sigma$ is connected.

It has been shown that in any space satisfying axioms 0 and $C$ (1) if $M$ is a separable point set, $M$ is completely separable, and (2) if $M$ is separable, any subset of $M$ is separable.

In order to show that any two points of $S$ lie in a separable continuum, suppose first that $P$ and $Q$ are two points of $S$. Obviously, $\left(B_{1}^{*}+B_{2}^{*}+\cdots\right)$ is separable and connected, and therefore $W+\left(B_{1}^{*}+\right.$ $\left.B_{2}^{*}+\cdots\right)$ is a separable continuum. In case $P$ and $Q$ both lie in $W+\left(B_{1}^{*}+B_{2}^{*}+\cdots\right)$, this continuum has the desired properties. In case $P$ does not belong to this set, $P$ belongs to $i_{x}[I(y)]$ for some $y$ in $C^{\prime}$. Let $M_{P}$ be the set to which point $R$ belongs if and only if, either

(1) there is a finite positive integer sequence $x_{1}, x_{2}, \cdots, x_{n}$ such that $R$ belongs to $i_{x_{1}}\left[i_{x_{2}}\left[\cdots i_{x_{n}}\left[i_{x}(I(y))\right] \cdots\right]\right]$, or

(2) there is a positive integer $q$ such that $R$ belongs to $i_{q}\left[i_{x}(I(y))\right]$, or

(3) $R$ belongs to $i_{x}[I(y)] . \quad M_{P}+\left(B_{1}^{*}+B_{2}^{*}+\cdots\right)+W$ is a separable continuum. If $Q$ does not belongs to this set, let $M_{Q}$ be a set related to $Q$ like $M_{P}$ was related to $P$. The continuum $M_{P}+M_{Q}+\left(B_{1}^{*}+\right.$ $\left.B_{2}^{*}+\cdots\right)+W$ is separable.

The statement that $\Sigma$ is locally separable at the point $P$ means that there is a region $R$ containing $P$ such that $R$ is separable. Alexandroff [1] has shown that if $\beta$ is a connected, locally completely separable, 
space satisfying axioms 0 and $C$, then $\beta$ is completely separable. It is interesting to note that $\Sigma$ is locally separable, and therefore locally completely separable, at each point except those of a separable set, and yet, $\Sigma$ is not separable.

$\Sigma$ is obviously locally separable at all points not belonging to $W$. Since every region that contains a point of $W$ contains uncountably many mutually exclusive domains, $\Sigma$ is not locally separable at any point of $W$. Furthermore $\left(B_{1}^{*}+B_{2}^{*}+\cdots\right)$ is separable, and so $\overline{\left(B_{1}^{*}+B_{2}^{*}+\cdots\right)}$ is separable, and thus, since $W$ is a subset of the latter, $W$ is separable.

$\Sigma$ is said to be semi-locally-connected [5] at point $P$ if and only if it is true that if $R$ is a region containing $P, R$ contains a region $R^{\prime}$ containing $P$ such that $S-R$ does not intersect infinitely many components of $S-R^{\prime} . \quad \Sigma$ is said to be semi-locally-connected if and only if $\Sigma$ is semi-locally-connected at each point.

The space $\Sigma$ is obviously semi-locally-connected because $S$ minus any region has only a finite number of components.

\section{PART 2}

Suppose that $\Sigma$ is a space satisfying the conditions specified on the first page of this paper.

For each positive integer $j$ let $G_{j}$ denote the collection of all open sets which have diameter less that $j^{-1}$.

Let $P$ denote some definite point, and suppose $n$ is a positive integer such that no countable subcollection of $G_{n}$ covers $S$. Let $R_{n}$ be some region of $G_{n}$ which contains $P$, let $H_{1}=\left\{R_{n}\right\}$, and let $K_{1}$ be the boundary of $R_{n}$.

For each point $Q$ of $S$ let $\Delta(Q)$ be the least integer $j>n$ such that some region $R(Q)$ of $G_{n}$ contains every region of $G_{j}$ that intersects a region of $G$, that contains $Q$.

It has been shown that in a space satisfying these axioms if $L$ is a separable point set and $G$ is a collection of open sets covering $L$, then some countable subcollection of $G$ covers $L$. Therefore, there is a countable point set $T_{1}$ dense in $K_{1}$ such that the collection $H_{2}$ of all $R(Q)$ 's, for Q's in $T_{1}$, covers $K_{1}$. Let $K_{2}$ be the sum of the boundaries of all the sets in $H_{1}+H_{2}$. There is a countable point set $T_{2}$ dense in $K_{2}$ such that the collection $H_{3}$ of all $R(Q)$ 's for $Q$ 's in $T_{2}$, covers $K_{2}$. Let $K_{3}$ be the sum of the boundaries of the sets in $H_{1}+H_{2}+H_{3}$, and so forth.

There is a point $B$ not in the closure of $H=\left(H_{1}+H_{2}+\cdots\right)^{*}$. Let $M$ be a compact continuum containing $P$ and $B$.

Case 1. Suppose some point $A$ of $M-M \cdot H$ is a limit point of $K=K_{1}+K_{2}+\cdots$. 
Let $R_{1}^{\prime}$ be a region of $G_{n}$ containing $A$, let $Q_{1}$ be a point of $T=$ $T_{1}+T_{2}+\cdots$ in $R_{1}^{\prime}$, and let $x_{1}$ be the largest integer $i$ such that $R_{1}^{\prime}$ belongs to $G_{i}$. Let $R_{2}^{\prime}$ be a region of $C_{x_{1+1}}$ containing $A$ such that $\bar{R}_{2}^{\prime}$ lies in $R_{1}^{\prime}-Q_{1}$. Let $Q_{2}$ be a point of $T$ in $R_{2}^{\prime}$ and let $x_{2}$ be the largest integer $i$ such that $R_{2}^{\prime}$ is in $G_{i}$. Obtain $R_{3}^{\prime}, Q_{3}$, and $x_{3}$ similarly, and so forth. $n \leqq x_{1}<x_{2}<x_{3}<\ldots$. For each $i, \Delta\left(Q_{i}\right)>x_{i}$. Otherwise, for some $i, R\left(Q_{i}\right)$ would contain $R_{i}^{\prime}$, and thus $A$. However, there is a positive integer $t>n$ such that if $x, y$, and $z$ are regions of $G_{t}$ such that $x \cdot y$ and $y \cdot z$ exist and $x$ contains $A$, then $R_{n}$ contains $x+y+z$. For some $s>t, \Delta\left(Q_{s}\right)>t$. But $R_{n}$ contains every region of $G_{t}$ that intersects a region of $G_{t}$ that contains $G_{s}$. So $\Delta\left(Q_{s}\right) \leqq t$, which is a contradiction.

Case 2. Suppose no point of $M-M \cdot H$ is a limit point of $K$. For each point $Q$ of $M-M \cdot H$ let $g_{Q}$ be a region containing $Q$ such that $g_{Q}$ contains no point of $K+P$. Some finite subcollection $C$ of the $g_{Q}$ 's covers this set of limit points. Let $D=H-H \cdot \bar{C}^{*}$. Let $C_{1}$ be the component of $M-M \cdot \bar{D}$ which contains $B$. Some point $z$ of $M \cdot \bar{D}$ is a limit point of $C_{1}$. But $z$ lies in a region $r$ of $H$, and therefore $C_{1}$ would intersect the boundary of $r$, and thus contain a limit point of $K$. This yields a contradiction.

Since, for each $n$, some countable subcollection of $G_{n}$ covers $S, \Sigma$ is completely separable.

\section{REFERENCES}

1. Paul Alexandroff, Uber die Metrization der im Kleinen Kompakten topologischen Raume, Mathematische Annalen, 92 (1924) 294-301.

2. F. B. Jones, On a property related to separability in metric spaces, Journal of the Elisha Mitchell Scientific Society, 70 (1954), 30-33.

3. - A theorem concerning locally peripherally separable spaces, Bull. Amer. Math. Soc. 41 (1935), 437-439.

4. R. L. Moore, Foundations of point set theory, Amer. Math. Soc. Colloq. Publ., vol 13 1932.

5. G. T. Whyburn, Semi-locally-connected sets, Amer. J. Math. 61 (1939), 733-749.

The UNIVERSITY OF TEXAS 


\section{PACIFIC JOURNAL OF MATHEMATICS}

\section{EDITORS}

David Gilbarg

Stanford University

Stanford, California

F. H. Brownell

University of Washington

Seattle 5, Washington
A. L. Whiteman

University of Southern Californıa Los Angeles 7, California

L. J. PaIge

University of California

Los Angeles 24, California

\section{ASSOCIATE EDITORS}
E. F. BECKENBACH
T. M. CHERRY
D. DERRY

E. HEWITT
A. HORN
L. NACHBIN
M. OHTSUKA

H. L. ROYDEN

M. M. SCHIFFER
E. SPANIER

E. G. STRAUS

F. WOLF

\section{SUPPORTING INSTITUTIONS}

\author{
UNIVERSITY OF BRITISH COLUMBIA \\ CALIFORNIA INSTITUTE OF TECHNOLOGY \\ UNIVERSITY OF CALIFORNIA \\ MONTANA STATE UNIVERSITY \\ UNIVERSITY OF NEVADA \\ NEW MEXICO STATE UNIVERSITY \\ OREGON STATE COLLEGE \\ UNIVERSITY OF OREGON \\ OSAKA UNIVERSITY \\ UNIVERSITY OF SOUTHERN CALIFORNIA
}

\author{
STANFORD UNIVERSITY \\ UNIVERSITY OF TOKYO \\ UNIVERSITY OF UTAH \\ WASHINGTON STATE COLLEGE \\ UNIVERSITY OF WASHINGTON \\ AMERICAN MATHEMATICAL SOCIETY \\ CALIFORNIA RESEARCH CORPORATION \\ HUGHES AIRCRAFT COMPANY \\ SPACE TECHNOLOGY LABORATORIES \\ NAVAL ORDNANCE TEST STATION
}

Mathematical papers intended for publication in the Pacific Journal of Mathematics should be typewritten (double spaced), and the author should keep a complete copy. Manuscripts may be sent to any one of the four editors. All other communications to the editors should be addressed to the managing editor, L. J. Paige at the University of California, Los Angeles 24, California.

50 reprints per author of each article are furnished free of charge; additional copies may be obtained at cost in multiples of 50 .

The Pacific Journal of Mathematics is published quarterly, in March, June, September, and December. The price per volume (4 numbers) is $\$ 12.00$; single issues, $\$ 3.50$. Back numbers are available. Special price to individual faculty members of supporting institutions and to individual members of the American Mathematical Society: $\$ 4.00$ per volume; single issues, $\$ 1.25$.

Subscriptions, orders for back numbers, and changes of address should be sent to Pacific Journal of Mathematics, 2120 Oxford Street, Berkeley 4, California.

Printed at Kokusai Bunken Insatsusha (International Academic Printing Co., Ltd.), No. 6, 2-chome, Fujimi-cho, Chiyoda-ku, Tokyo, Japan.

PUBLISHED BY PACIFIC JOURNAL OF MATHEMATICS, A NON-PROFIT CORPORATION

The Supporting Institutions listed above contribute to the cost of publication of this Journal, but they are not owners or publishers and have no responsibility for its content or policies. 


\section{Pacific Journal of Mathematics}

\section{Vol. 10, No. $2 \quad$ October, 1960}

Maynard G. Arsove, The Paley-Wiener theorem in metric linear spaces ........

Robert (Yisrael) John Aumann, Acceptable points in games of perfect

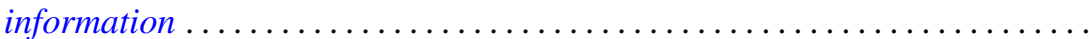

A. V. Balakrishnan, Fractional powers of closed operators and the semigroups generated by them ... . . . . . . . . . . . . . . . . . . . . . . . . . . . . 419

Dallas O. Banks, Bounds for the eigenvalues of some vibrating systems . . . . . 439

Billy Joe Boyer, On the summability of derived Fourier series . . . . . . . . . . . 475

Robert Breusch, An elementary proof of the prime number theorem with

remainder term ...................................

Edward David Callender, Jr., Hölder continuity of $n$-dimensional



L. Carlitz, Note on Alder's polynomials ......................... 517

P. H. Doyle, III, Unions of cell pairs in $E^{3} \ldots \ldots \ldots \ldots \ldots \ldots \ldots \ldots \ldots \ldots \ldots \ldots . \ldots 21$

James Eells, Jr., A class of smooth bundles over a manifold . . . . . . . . . . . . 525

Shaul Foguel, Computations of the multiplicity function . . . . . . . . . . . . . . 539

James G. Glimm and Richard Vincent Kadison, Unitary operators in

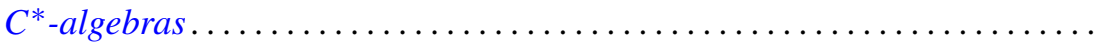

Hugh Gordon, Measure defined by abstract $L_{p}$ spaces . . . . . . . . . . . 557

Robert Clarke James, Separable conjugate spaces ....................

William Elliott Jenner, On non-associative algebras associated with bilinear forms

Harold H. Johnson, Terminating prolongation procedures

John W. Milnor and Edwin Spanier, Two remarks on fiber homotopy type .

Donald Alan Norton, A note on associativity . .

Ronald John Nunke, On the extensions of a torsion module.

Joseph J. Rotman, Mixed modules over valuations rings . . . . .

A. Sade, Théorie des systèmes demosiens de groupoï des . .

Wolfgang M. Schmidt, On normal numbers . .

661

Berthold Schweizer, Abe Sklar and Edward Oakley Thorp, The metrization of

statistical metric spaces

John P. Shanahan, On uniqueness questions for hyperbolic differential

equations

A. H. Stone, Sequences of coverings

Edward Oakley Thorp, Projections onto the subspace of compact operators

L. Bruce Treybig, Concerning certain locally peripherally separable spaces

Milo Wesley Weaver, On the commutativity of a correspondence and a

permutation

David Van Vranken Wend, On the zeros of solutions of some linear complex

differential equations. 